\title{
THE BOUNDARY OF A SIMPLY CONNECTED DOMAIN ${ }^{1}$
}

\section{GEORGE PIRANIAN}

1. Prime ends. The foundation for the study of boundaries of simply connected domains in the plane was laid by Carathéodory [2], who defined ends in general and prime ends in particular, and who classified prime ends into four kinds. To facilitate a brief survey of the subject of prime ends, I introduce a few definitions concerning a simply connected domain $B$. In my definitions, I follow essentially the work of Caratheodory, except that, for the sake of brevity, I omit the description of ends in general and aim directly at prime ends.

A sequence $K$ of crosscuts $c_{n}$ of $B$ is a chain provided

(i) the diameter of $c_{n}$ tends to 0 as $n \rightarrow \infty$;

(ii) for each index $n$, the set $\bar{c}_{n} \cap \bar{c}_{n+1}$ (where the bar indicates closure) is empty;

(iii) some fixed point $O$ in $B$ cannot be joined to any crosscut $c_{n}(n>1)$ by any path in $B$ which does not meet the crosscut $c_{n-1}$.

Two chains $K=\left\{c_{n}\right\}$ and $K^{\prime}=\left\{c_{n}^{\prime}\right\}$ in $B$ are equivalent provided each crosscut $c_{n}$ effects a separation, relative to $B$, of the point $O$ from all except finitely many of the crosscuts $c_{n}^{\prime}$.

An equivalence class of chains in $B$ is a prime end of $B$.

If $P$ is a prime end of $B$, let $K=\left\{c_{n}\right\}$ denote a chain which belongs to $P$, and for each index $n$ let $B_{n}$ denote that subdomain of $B$ which is determined by $c_{n}$ and does not contain the point $O$. The set $I(P)=\bigcap \bar{B}_{n}$ will be called the impression of $P$.

It should be remarked that Caratheodory and some other writers applied the term prime end to the point set $I(P)$, but that they regarded as distinct two prime ends $P_{1}$ and $P_{2}$ corresponding to two nonequivalent chains, even in cases where the two sets $I\left(P_{1}\right)$ and $I\left(P_{2}\right)$ are identical. The distinction between a prime end and its impression formalizes the ideas which are involved.

Caratheodory's principal theorem on the correspondence between boundaries under conformal mappings $[2$, p. 350] can be expressed as follows: If $f(z)$ maps the unit disk conformally and one-to-one onto the

An address delivered before the Evanston meeting of the Society on November 23, 1956, by invitation of the Committee to Select Hour Speakers for Western Sectional Meetings; received by the editors March 20, 1957.

1 The address was prepared under Contract DA 20-018-ORD-13585 with the Office of Ordnance Research, U. S. Army. 
domain $B$, it induces a one-to-one mapping between the points of the unit circle and the prime ends of $B$.

2. Alternate definitions, and extensions. Koebe [10, pp. 217-219] introduced an alternate definition of prime ends (boundary elements). It is based on equivalence classes of paths to accessible boundary points. Two boundary paths $\Gamma_{1}$ and $\Gamma_{2}$ in $B$ (that is, two homeomorphic images $p_{i}=p_{i}(t)(i=1,2)$ of the open unit interval) are equivalent if they both approach the same boundary point $p(1)$ as $t \rightarrow 1$ and if, for each $\epsilon>0$, every point $p_{1}(t)\left(t>t_{\epsilon}\right)$ can be joined to $\Gamma_{2}$ by a path which lies in $B$ and has diameter less than $\epsilon$. Prime ends correspond to "Dedekind sections" in the space of equivalence classes of paths to accessible boundary points.

Ursell and Young [16, see (4.5) and (4.6) on p. 5] took an approach intermediate between that of Caratheodory and that of Koebe. Their principal contributions are the study of the fine structure of the individual prime end, and the investigation of the relation between prime ends of $B$ and the interior of the complement of $B$.

It is natural that the concept of prime ends should be subjected to axiomatization, and that it should be extended to domains of infinite connectivity, to domains in euclidean three-space, and finally to abstract spaces. In this direction, papers by Kaufmann [9], Mazurkiewicz [13], and Freudenthal [6] must be mentioned. Suvorov [15] defined prime ends of a sequence of domains converging to a nucleus. But these are developments which lead away from my problem, and I return to the ideas of Caratheodory.

3. The classification of prime ends. A point $p$ in the impression $I(P)$ is a principal point (relative to $P$ ) provided every neighborhood of $p$ contains a crosscut of a chain which belongs to $P$; otherwise, it is a subsidiary point (relative to $P$ ). Elementary considerations show that every impression $I(P)$ contains at least one point which is a principal point relative to $P$.

A prime end is of the first kind if its impression consists of a single point, which is then necessarily a principal point. In other words, $P$ is of the first kind provided

(a) $I(P)$ contains only one principal point, and

(b) $I(P)$ contains no subsidiary points.

A prime end is of the second, third, or fourth kind if it satisfies only condition (a), only condition (b), or neither of the two conditions, respectively.

For the sake of brevity, we shall also say that a point set on the boundary of $B$ is an impression of the first, second, third, or fourth 
kind if it is the impression of a prime end of the corresponding kind.

By way of illustration, we consider the domain suggested by Figure 1. The domain consists of the interior of a square from which various line segments have been deleted. Each impression on the upper edge $A C$ of the square is of the first kind; the same is true of impressions lying on slits that issue from the upper edge. The point $B$ is the impression of infinitely many prime ends.

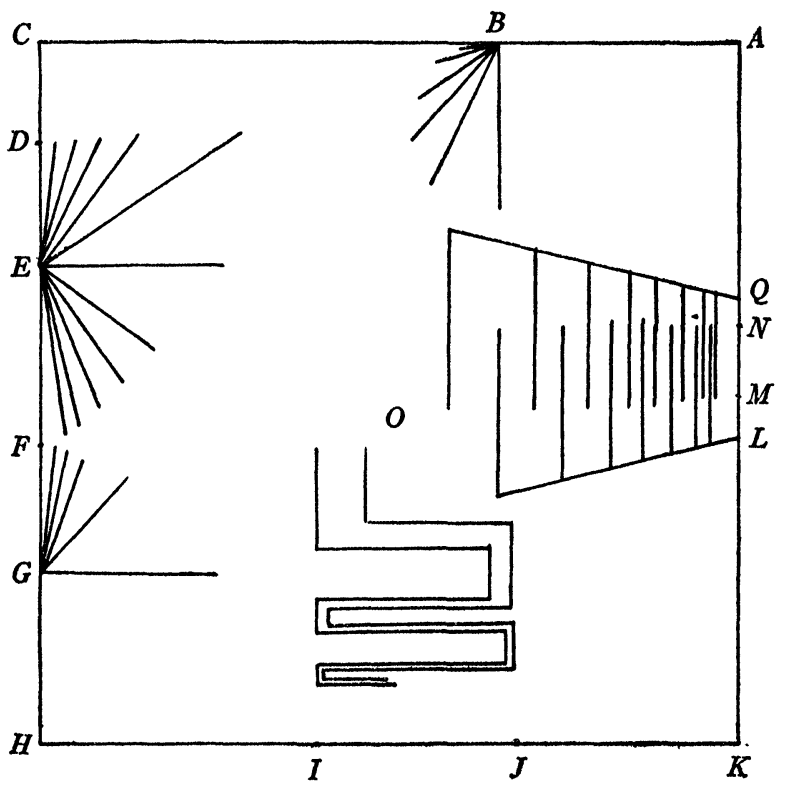

FIG. 1

Each impression on the side $\mathrm{CH}$ or on one of the slits issuing from $\mathrm{CH}$ is of the first or second kind. In particular, there are two impressions of the second kind, namely the segments $D E$ (with $D$ as principal point) and $E G$ (with $F$ as principal point). The point sets consisting of $E$ and $G$, respectively, are the impressions of infinitely many prime ends of the first kind.

On $H K$, there are two impressions of the second kind, namely the segment $I J$ with $I$ as principal point, and the same segment with $J$ as principal point. In addition, the segment $I J$ is an impression of the third kind. If the figure is modified by adjoining infinitely many further slits (following closely the slits that approach the segment $I J$ ), then $I J$ becomes the impression of infinitely many prime ends of the third kind. 
On $K A$ and the adjoining slits, all impressions are of the first kind, except that the segment $L Q$ is an impression of the fourth kind. The points on the closed segment $M N$ are principal points; the points on the remainder of the closed segment $L Q$ are subsidiary points. The two point sets consisting of $L$ and $Q$, respectively, are impressions of the first kind.

4. The distribution problem. Urysohn observed $[17$, p. 235] that the one-to-one correspondence between the unit circle and the set of prime ends of a simply connected domain $B$ induces a Hausdorff topology in the set of prime ends of $B$. We denote by $U(B)$ the topological space of prime ends of $B$, and by $U_{k}=U_{k}(B)(k=1,2,3,4)$ the set of prime ends in $U(B)$ which are of the $k$ th kind. A question of Carathéodory $[2, \mathrm{p} .325]$ can now be phrased as follows: Is it possible that $U_{2}(B)=U(B)$ ?

This question was answered in the negative by Weniaminoff [18], who applied Lindelöf's theorem to characterize the prime ends of the first and second kinds in terms of the radial behavior of the mapping function $f(z)$ which carries the unit disk onto $B$. The function $f(z)$ carries a point $e^{i \theta}$ into a prime end of the first or second kind if and only if the radial limit $f\left(e^{i \theta}\right)$ of $f(z)$ exists. This implies that if the sets $U_{3}(B)$ and $U_{4}(B)$ are empty, the function $f\left(e^{i \theta}\right)$ exists everywhere. Also, $f\left(e^{i \theta}\right)$ is then of Baire class 1, and its set of discontinuities is therefore of first category. Finally, the set $U_{2}(B)$ is the image of the set of discontinuities of $f\left(e^{i \theta}\right)$, and therefore $U_{2}(B)$ is also of first category. Essentially the same argument was used, independently, by van Aardenne-Ehrenfest and Wolff [1]. Another solution of the Carathéodory problem is due to Urysohn [17].

The argument of Weniaminoff actually yields more that I have stated so far: If the sets $U_{3}$ and $U_{4}$ are empty, then $U_{2}$ is of first category and of type $F_{\sigma}$. Lohwater and I have proved a converse of this [12]: Let $E_{2}$ be any set on the unit circle $C$, of type $F_{\sigma}$ and of first category. Then there exists a function $f(z)$, bounded and schlicht in $D$, which carries each point of $E_{2}$ into a prime end of the second kind, and each point of $C-E_{2}$ into a prime end of the first kind.

The fact that Carathéodory's special question on the set $U_{2}$ is amenable to such complete treatment constitutes a temptation to propose the following problem.

PROBLEM 1. To find necessary and sufficient conditions on the decomposition of the unit circle $C$ into four disjoint sets $E_{k}(k=1,2,3,4)$ in order that, under some conformal mapping of the unit disk onto a bounded schlicht domain, the points of each set $E_{k}$ correspond to prime ends of the kth kind. 
The solution of this general problem will be vastly more difficult than the solution of the special problem where $U_{3}$ and $U_{4}$ are empty. The difference is not merely one of degree, but it goes very deep; for the solution of the special problem can be stated in terms of topological concepts alone, while Fatou's theorem [5, p. 337] implies that the point sets $E_{3}$ and $E_{4}$ are subject to metric restrictions. Pending the development of methods and skills which are not available today, it seems advisable to replace Problem 1 by a less forbidding version:

Problem 2. To find necessary and sufficient conditions on the decomposition of $C$ into four disjoint sets $E_{k}(k=1,2,3,4)$ in order that, for some simply connected domain $B$ and some appropriate homeomorphism between $C$ and $U(B)$, each set $E_{k}$ corresponds to the set $U_{k}(B)$.

5. Necessary conditions on the decomposition. Throughout this section, $B$ denotes a fixed (but arbitrary) simply connected domain; $P$ denotes a prime end of $B$, and $|P|$ the diameter of $P$, defined as the diameter of its impression $I(P)$.

THEOREM 1. For every positive number $h$, the set of prime ends of diameter at least $h$ is closed.

Corollary. The set $U_{1}(B)$ is of type $G_{\delta}$.

Proof. Let $\left\{B_{n}\right\}$ be a chain of domains corresponding to $P$, and suppose that every neighborhood of $P$ (in the sense of Urysohn) contains a prime end of diameter at least $h$. Then every set $\bar{B}_{n}$ contains a subset of diameter at least $h$, and therefore $|P| \geqq h$. The corollary follows since $U_{1}(B)=\cap M_{n}$, where $M_{n}$ denotes the set of prime ends of diameter less than $1 / n(n=1,2, \cdots)$.

For the further analysis, we need the concept of cluster sets. A complex number $w$ belongs to the (complete) cluster set of the function $f(z)$ at $e^{i \theta}$ provided there exists a sequence of points $z_{n}$ in the unit disk $D$, converging to $e^{i \theta}$, and having the property that $f\left(z_{n}\right) \rightarrow w$ as $n \rightarrow \infty$. The point $w$ belongs to the radial cluster set of $f(z)$ at $e^{i \theta}$, if the points $z_{n}$ can be chosen on the radius of $e^{i \theta}$.

If $f$ maps the unit disk $D$ conformally onto $B$ in such a way that the center of $D$ is carried to the point $O$ in $B$, and if $e^{i \theta}$ is the point corresponding to the prime end $P$ with the chain $\left\{c_{n}\right\}$, then the image of the radius of $e^{i \theta}$ meets each of the cuts $c_{n}$. It follows that every principal point of $P$ belongs to the radial cluster set of $f$ at $e^{i \theta}$. On the other hand, Lindelöf has shown [11, p. 28] (see also Montel [14, pp. 48-52] and Gross [7, p. 254]) that the radial cluster set at $e^{i \theta}$ contains no subsidiary points of $P$. It follows that all points $e^{i \theta}$ 
at which the radial limit exists correspond to prime ends of the first or second kind, and that all points $e^{i \theta}$ at which the radial cluster set coincides with the complete cluster set correspond to prime ends of the first or third kind.

THEOREM 2. On every interval of $U(B)$, the set $U_{1} \cup U_{2}$ has the power of the continuum.

This proposition follows immediately from Fatou's theorem. It can also be proved directly by showing that every interval of impressions of prime ends contains $2^{\aleph_{0}}$ points that are appropriately accessible from the interior of $B$.

TheOREM 3. The set $U_{3} \cup U_{4}$ is of type $G_{\delta \sigma}$.

The theorem follows from the fact that the set of radii on which $f$ has positive oscillation is a set of type $G_{\delta \sigma}$ (see Hausdorff $[8$, p. 273]).

Before closing this section, we call attention to a recent result of Collingwood [3] which implies that if the function $f$ is meromorphic in $D$, then there exists a residual set $E$ on $C$ such that, at each point $e^{i \theta}$ in $E$, the radial cluster set at $e^{i \theta}$ coincides with the complete cluster set at $e^{i \theta}$. It follows (see Collingwood [4, p. 349]) that for every simply connected domain $B$ the set $U_{1} \cup U_{3}$ is residual.

6. The case where $U_{4}$ is empty. The results of the preceding section, together with an appropriate geometrical construction, lead to the following partial solution of the second distribution problem.

THEOREM 4. Let $B$ be a simply connected domain without prime ends of the fourth kind. Then there exist two sequences of sets $F_{n}$ and $M_{n}$ in the space $U(B)$ which have the following three properties.

1. The sets $F_{n}$ are mutually disjoint, and the set $\mathrm{U}_{n=1}^{m} F_{n}$ is closed, for every $m$.

2. For each $n$, the set $M_{n}$ is of type $G_{\delta}$ and is contained in $F_{n}$; also, on every open subset of $F_{n}$, both $M_{n}$ and its complement have the power of the continuum.

3. $U_{1}=U-\mathrm{U} F_{n}, U_{2}=\mathrm{U}\left(F_{n}-M_{n}\right), U_{3}=\mathrm{U} M_{n}$.

On the other hand, let $\left\{F_{n}\right\}$ and $\left\{M_{n}\right\}$ be two sequences of sets on $C$ such that Conditions 1 and 2 are satisfied, and let

$$
E_{1}=C-U F_{n}, \quad E_{2}=U\left(F_{n}-M_{n}\right), \quad E_{3}=U M_{n} .
$$

Then there exists a domain $B$ such that some homeomorphism between $C$ and $U(B)$ carries each of the sets $E_{k}$ into the corresponding set $U_{k}(B)$ $(k=1,2,3)$.

To prove the first part of the theorem, we let $F_{1}$ denote the set of 
prime ends $P$ with $|P| \geqq 1$, and for $n=2,3, \cdots$ we let $F_{n}$ denote the set of prime ends $P$ with $1 /(n-1)>|P| \geqq 1 / n$. Condition 1 and the first part of Condition 3 are then satisfied, by Theorem 1 .

Let $f$ denote a function which maps the unit disk $D$ conformally onto $B$, and let $E_{n j}$ be the set of points $e^{i \theta}$ for which there exist two values $r_{1}$ and $r_{2}\left(1-2^{-i}<r_{1}<r_{2}<1\right)$ such that

$$
\left|f\left(r_{1} e^{i \theta}\right)-f\left(r_{2} e^{i \theta}\right)\right|>1 / n-1 / 2^{i} .
$$

Then $E_{n j}$ is open, and therefore the set $E_{n}=\bigcap_{j=1}^{\infty} E_{n j}$ is of type $G_{\delta}$. Now let $M_{n}^{*}$ be the set of prime ends of $B$ which correspond to points in $E_{n}$. Then each prime end in $M_{n}^{*}$ lies in $\bigcup_{m=1}^{n} F_{m}$, by virtue of its diameter. Since $E_{n}$ consists of all points of $C$ on whose radius the function $f$ has oscillation of amplitude at least $1 / n$, and since $U_{4}$ is empty (by hypothesis), $M_{n}^{*}$ consists of all prime ends of the third kind and of diameter at least $1 / n$.

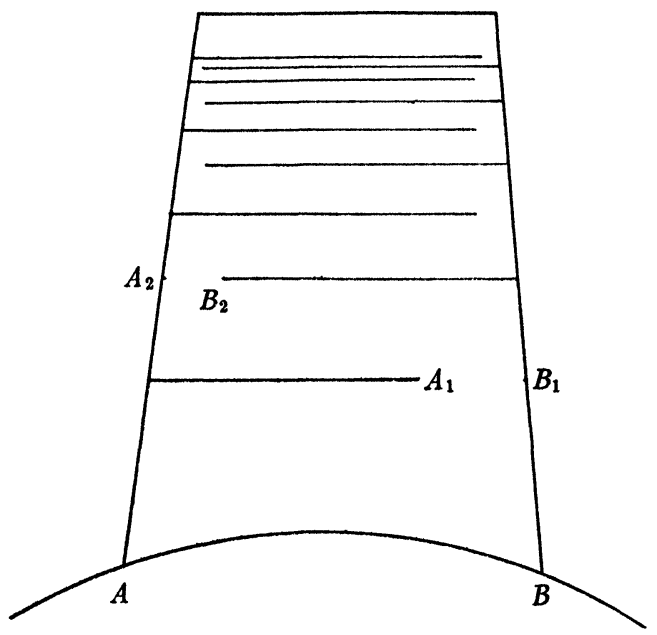

FIG. 2

Let $M_{n}=M_{n}^{*} \cap F_{n}$. The first part of Condition 2 is clearly satisfied; the second part follows from the theorem of Weniaminoff and the fact that a dense set of type $G_{\delta}$ is not denumerable.

7. The Carathéodory boxes. To complete the proof of the theorem, we use a device which is a slight modification of a construction described by Carathéodory [ 2 ; see the figure on p. 366 and the discussion on p. 369].

Let $\left\{F_{n}\right\}$ and $\left\{M_{n}\right\}$ be two sequences of point sets on $C$, subject to Conditions 1 and 2 of Theorem 4 . Then each set $M_{n}$ can be repre- 


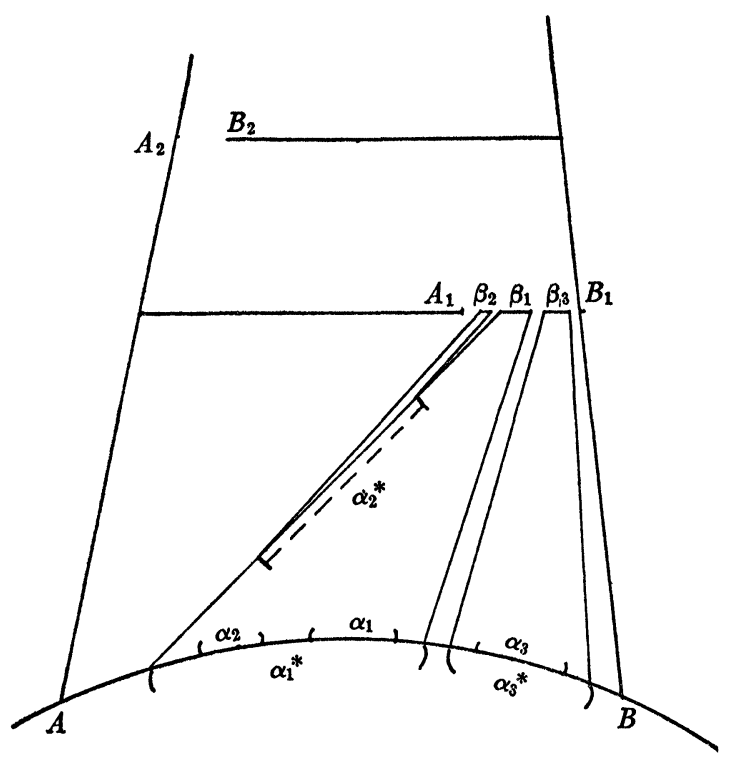

FIG. 3

sented as the intersection of a decreasing sequence of open sets $G_{n k}$. We confine our attention to a single one of the open $\operatorname{arcs} A B$ constituting the set $G_{11}$. With this arc as base we construct a "trapezoid" lying outside of $C$ (see Figure 2). Inside of the trapezoid we draw infinitely many rectilinear crosscuts, each parallel to the base opposite $A B$, and with the distance between the $j$ th crosscut and that base tending to 0 decreasingly, as $j \rightarrow \infty$. From the $j$ th crosscut we delete a short segment $A_{j} B_{j}$ at the right or left end, according as $j$ is odd or even. And we imagine the arc $A B$ mapped onto each of the deleted segments $A_{j} B_{j}$, by any homeomorphism which carries the point $A$ into $A_{j}$.

By Condition 2 in the theorem, there exists in the closure of $A B$ a perfect set which lies in the complement of $M_{1}$ and which contains both the points $A$ and $B$. We choose such a set and denote it by $N_{0}$. Without loss of generality, we may assume that each of the open arcs of $A B$ contiguous to $N_{0}$ contains at least one point of $M_{1}$; for any arc which fails in this respect can simply be incorporated into $N_{0}$.

Let $\alpha_{1}, \alpha_{2}, \cdots$ denote the open arcs that compose the set $A B-N_{0}$. We erect a trapezoid which has for one side the image $\beta_{1}$ of $\alpha_{1}$ on $A_{1} B_{1}$, and for the opposite side an $\operatorname{arc} \alpha_{1}^{*}$ on $A B$ which contains $\alpha_{1}$ and whose end points are essential points of $N_{0}$ (see Figure 3; by an essential point of $N_{0}$ we mean a point of $N_{0}$ which is not an 
end point of any arc of the complement of $N_{0}$ ). We imagine the two segments of $\alpha_{1}^{*}$ which are complementary to $\alpha_{1}$ mapped onto the corresponding sides of the quadrilateral which has just been erected.

If the arc $\alpha_{2}$ does not lie on $\alpha_{1}$, we accord it similar treatment. If it lies on $\alpha_{1}$, we give it a quadrilateral one of whose sides is the image $\beta_{2}$ of $\alpha_{2}$ on $A_{1} B_{1}$, while the opposite side is a segment $\alpha_{2}^{*}$ on the corresponding side of the quadrilateral over $\alpha_{1}$; the segment $\alpha_{2}^{*}$ must contain the image of $\alpha_{2}$, and its endpoints must be images of essential points of $N_{0}$.

We continue thus, with the proviso that no segment $\alpha_{k}^{*}$ shall have in its interior or as one of its endpoints any vertex of a previously constructed quadrilateral.

After each of the arcs $\alpha_{k}$ has been treated in this manner, we delete the $\operatorname{arcs} \alpha_{k}^{*}$. Now the unit disk, together with certain extensions that reach to the segment $A_{1} B_{1}$, constitutes a simply connected domain. And the open subset $\bigcup \alpha_{k}$ of $G_{11}$, which contains the set $M_{1} \cap A B$, has been mapped onto the open set $U \beta_{k}$ on $A_{1} B_{1}$.

Next we consider the intersection of this open subset of $A_{1} B_{1}$ with the image of $G_{12} \cap A B$ on $A_{1} B_{1}$. From the closure of each component of this intersection we delete a perfect set $N_{1}$ which contains both endpoints of the component and whose pre-image does not meet the set $M_{1}$. We then repeat our construction with the open $\operatorname{arcs} \alpha_{k}^{(1)}$ that remain; that is, for each of these open arcs we construct a quadrilateral one of whose sides is the image $\beta_{k}^{(1)}$ on $A_{2} B_{2}$ of the arc.

The result of the continuation of the process is called a Caratheodory box. It is easily seen that the unit disk together with the Carathédory box over $A B$ is a simply connected domain.

Consider now any chain $K=\left\{c_{n}\right\}$ of crosscuts which have at least one end in the Carathéodory box. Either the crosscuts $c_{n}$ approach the upper edge, that is, the lid of the box, in which case $K$ belongs to a prime end $P$ of the third kind, and $I(P)$ coincides with the lid; or the crosscuts approach a point which is not on the lid. To analyze the latter case, we observe that every quadrangle which is erected during the construction is ultimately supplied with extensions which reach to the lid of the box; that every point which is a vertex of a quadrilateral is a limit point of such vertices; and that every vertex of a quadrilateral is a boundary point of the domain. If a chain of crosscuts approaches a point $p$ which is a limit point of vertices of quadrilaterals and does not lie on the lid, then the chain belongs to a prime end $P$ of the second kind; $p$ is the principal point of $P$; and $I(p)$ consists of an infinite "polygonal line" and of the lid of the box.

It follows that there exists a homeomorphism between the unit 
circle and the space of prime ends of our domain which maps into prime ends of the third kind all points on $M_{1} \cap A B$; into prime ends of the second kind all limit points of this set which do not belong to the set; and into prime ends of the first kind all the remaining points of the circle.

It is easily seen how the same process can be applied to all other arcs of $G_{11}$. If the continuation is effected with uniformly high Carathéodory boxes, then the result is a domain which has prime ends of the third kind corresponding to all points of $M_{1}$, and prime ends of the second kind corresponding to all points of $\bar{M}_{1}-M_{1}$.

Meanwhile, the open circular arcs of $C-\bar{M}_{1}$ have been transformed into open continuous arcs. On these arcs we can erect "parallel quadrilaterals" (in the manner of $[12, \S 2]$ ) in such a way that all points of $F_{1}-M_{1}$ correspond to prime ends of the second kind.

The set $C-F_{1}$ is open, and its image consists of open continuous arcs. On these arcs we construct Carathéodory boxes corresponding to the set $M_{2}$, and further quadrilateral extensions corresponding to the set $F_{2}-\bar{M}_{2}$. The Carathéodory boxes and the extensions may be distorted; but they can be made uniformly large, except near the impressions of prime ends corresponding to points of $F_{1}$. The indefinite continuation of the process presents no difficulty; for after the completion of each stage, all further modifications have to be made on open, continuous arcs of the boundary. And while the process would be certain to drive any draftsman to despair, it yields a domain $B$ which constitutes a proof of the second part of Theorem 4 .

\section{REFERENCES}

1. T. van Aardenne-Ehrenfest and J. Wolff, Über die Grenzen einfachzusammenhängender Gebiete, Comment. Math. Helv. vol. 16 (1943-1944) pp. 321-323.

2. C. Carathéodory, Über die Begrenzung einfach zusammenhängender Gebiete, Math. Ann. vol. 73 (1913) pp. 323-370.

3. E. F. Collingwood, Sur le comportement a la frontière, d'une fonction méromorphe dans le cercle unité, C. R. Acad. Sci. Paris vol. 240 (1955) pp. 1502-1504. 349.

4. —_, A theorem on prime ends, J. London Math. Soc. vol. 31 (1956) pp. 344-

5. P. Fatou, Séries trigonométriques et séries de Taylor, Acta Math. vol. 30 (1906) pp. $335-400$. 210 .

6. H. Freudenthal, Enden und Primenden, Fund. Math. vol. 39 (1952) pp. 189-

7. W. Gross, Zum Verhalten analytischer Funktionen in der Umgebung singulärer Stellen, Math. Zeit. vol. 2 (1918) pp. 242-294.

8. F. Hausdorff, Mengenlehre, 3d ed., Berlin-Leipzig, 1935.

9. B. Kaufmann, Über die Berandung ebener und räumlicher Gebiete (Primendentheorie), Math. Ann. vol. 103 (1930) pp. 70-144. 
10. P. Koebe, Abhandlungen zur Theorie der konformen Abbildung, J. Reine Angew. Math. vol. 145 (1915) pp. 177-223.

11. E. Lindelöf, Sur un principe genéral de l'analyse et ses applications a la représentation conforme, Acta Societatis Scientiarum Fennicae. Nova Series A. vol. 46 (1920), no. 4 (1915).

12. A. J. Lohwater and G. Piranian, The boundary behavior of functions analytic in a disk, Annales Academiae Scientiarum Fennicae. Series A.I., no. 239, 1957, pp. 1-17.

13. S. Mazurkiewicz, Über die Definition der Primenden, Fund. Math. vol. 26 (1936) pp. 272-279.

14. P. Montel, Sur la représentation conforme, J. Math. Pures Appl. (7) vol. 3 (1917) pp. 1-54.

15. G. D. Suvorov, On the prime ends of a sequence of plane regions converging to a nucleus, Rec. Math. (Mat. Sbornik) N.S. 33(75) (1953) pp. 73-100; Amer. Math. Soc. Translations, ser. 2, vol. 1 (1955) pp. 67-93.

16. H. D. Ursell and L. C. Young, Remarks on the theory of prime ends, Memoirs of the American Mathematical Society, no. 3, 1951.

17. P. Urysohn, Über ein Problem von Herrn C. Carathéodory, Fund. Math. vol. 6 (1924) pp. 229-235.

18. V. Weniaminoff, Sur un problème de la représentation conforme de M. Carathéodory, Recueil Math. Soc. Math. Moscou vol. 31 (1922) pp. 91-93; Jahrb. Fortsch. Math. vol. 48 (1921-1922) p. 405.

UNIVERSITY OF Michigan 